outstrips the limited expertise available and therefore significant delays occur in treatment pathways. There is an urgent need to expand the skill set of the employed workforce and think 'outside the box'. A service run by clinical nurse specialists (CNS) can be an effective solution. CNS are already an integral part of multidisciplinary teams caring for a diverse range of patients including those with chronic conditions such as osteoporosis.

Objectives: We designed an innovative osteoporosis service with patients consulting only a metabolic bone CNS with a consultant rheumatologist providing remote oversight. The aim of the project was to improve the efficiency of the service by eliminating consultant appointments and reducing unnecessary hospital visits while continuing to deliver a high-quality and safe service.

Methods: A new pathway was implemented where a consultant rheumatologist and a CNS virtually triaged post menopausal women over the age of 65 into the service. A dedicated proforma provided the template for the CNS to undertake new patient telephone consultation. Relevant investigations were requested during the telephone clinic and treatment related information was despatched to help with shared decision making. All patients were then reviewed in a Rheumatologist-CNS virtual MDT. An appropriate parenteral treatment option was agreed and confirmed to each individual. The CNS worked through a safety checklist and provided further advice and support to the patient as necessary. Using the database, we compared the timelines for the patient journey to the conventional pathway, obtained the number of consultant follow-up appointments saved by implementing this service, and calculated total savings.

Results: In the pilot phase, 116 patients were triaged into the new service. The patient cohort was a combination of new referrals and patients taken from the consultants' waiting lists. The mean age of the participants was 78 years (65-93). The median time: from referral to virtual triage was 20 days $(0-308)$; from triage to new patient CNS telephone consultation was 20 days (0-137); and from virtual MDT to treatment authorisation was zero days (0-331 days). 45 patients had anabolic therapy commenced via home care. The remainder had anti resorptive therapy. No patient requested face-to-face review. Only one patient fed back that they would've preferred to see the consultant once. 116 new patient consultant appointments were saved and the median delay in treatment commencement was reduced from 84 to 38 days.

Conclusion: To our knowledge, this is the first successful example of an innovative service wholly provided by CNS for commencing parenteral anti-osteoporotic therapy with only remote consultant supervision. Our service redesign has significantly improved the efficiency of the parenteral osteoporosis pathway with reduction in treatment delay and a more streamlined patient journey. A nurse-delivered osteoporosis treatment pathway is highly effective, safe and provides an innovative solution to address thinly stretched health care resources for people with chronic conditions.

Disclosure of Interests: None declared

DOI: 10.1136/annrheumdis-2021-eular.1069

\section{POS1489-HPR TELEMEDICINE CONSULTATIONS IN RHEUMATOLOGY: IDENTIFIED PROBLEMS OF NEW TECHNOLOGY}

Y. Livshits ${ }^{1}$, O. Teplyakova ${ }^{1}$, A. Sarapulova ${ }^{1} .{ }^{1}$ Ural State Medical University, All Patient Department, Yekaterinburg, Russian Federation

Background: Telemedicine counseling (TMC) has gained rapid development during the COVID-19 pandemic. The prospect of using this technology in rheumatology was based on the possibility of getting maximum information about the patient during the survey, examination and interpretation of laboratory and instrumental data, that is excepting direct contact with the patient. Several rheumatological clinics have reported on the success of using TMC. However, there is very little data of the difficulties that can be encountered when organizing this process. Objectives: To characterize the identified problems during TMC in rheumatology, to suggest potential directions for their elimination.

Methods: Since June 2021, on the basis of the Medical Association "New Hospital", Yekaterinburg, Russian Federation, 76 TMCs have been performed on the profile of rheumatology in patients aged 29 to 71 years. Of these, 13 applied to the primary $\mathrm{TMC}$, the other patients were preliminarily examined in person. The consultation included the preliminary acquaintance with the examination results, a 20-minute video communication and writing of a conclusion. After each TMC, a survey was conducted between the doctor and the patient, including the identified deficiencies in counseling. The frequency of identified problems is presented as an absolute indicator and as a percentage of the total number of TMCs performed.

Results: We noted a high degree of patient satisfaction: 74 (97.4\%) responded that they received answers to all. However, according to the doctor, the following groups of problems were identified.

[1] Technical problems in 29 (38.2\%): most often there were various problems with the Internet, but there were also registered: the end of the charge on the patient's tablet, the patient was not registered in the electronic queue.
Elimination of these violations depends on the work of IT-specialists, but each consulting physician should be prepared for an immediate transition to an alternative form of communication (for example - telephone).

[2] Lack of objective examination, leading to the impossibility of correct remote diagnosis - $8(10.5 \%)$. This problem was identified due to the inability to establish the presence or absence of arthritis during the initial diagnosis (6 cases) and to clarify the nature of the rash (2 cases). All patients are invited for a face-to-face consultation.

[3] The need to write prescriptions for psychotropic drugs - 12 (15.8\%), which under the conditions of national legislation cannot be done in the TMC regime.

[4] The time spent directly on remote communication with the patient was 17.2 minutes (from 8 to 31 ), however, taking into account the study data and writing the conclusion, the total time was 40.7 minutes (from 21 to 73 ). Thus it turned out that the average time for remote and face-to-face consultations is the same, while TMC's payment is only about $50 \%$ of the face-to-face consultation. This situation reduces the doctor's interest in carrying out TMC The solution to the problem is associated with reducing the time for the documentation process through technical improvements. In addition, of the 9 patients in whom the TMC process lasted 60 minutes or more, 5 were diagnosed with fibromyalgia. It is possible that with a previously established diagnosis of fibromyalgia, only face-to-face counseling should be recommended to patients.

Conclusion: The TMC system is promising, however, there are a number of problems that need to be improved, since they can reduce the doctor's interest in using this technology.

Disclosure of Interests: None declared

DOI: 10.1136/annrheumdis-2021-eular.1183

\section{HPR Professional education, training and competencies}

\section{POS1490-HPR "WHERE DOES IT HURT?”: IDENTIFYING PAIN CONTENT AND ITS CONTEXT WITHIN PAEDIATRIC AND ADOLESCENT RHEUMATOLOGY TRAINING}

R. Lee $^{1,2}$, J. Mcdonagh ${ }^{1,2,3}$, M. Connelly ${ }^{4}$, S. Peters ${ }^{5}$, L. Cordingley ${ }^{6} .{ }^{1}$ Centre for Epidemiology Versus Arthritis, Centre for Musculoskeletal Research, Faculty of Biology, Medicine and Health, University of Manchester, Manchester, United Kingdom; ${ }^{2}$ NIHR Manchester Biomedical Research Centre, Manchester University NHS Foundation Trust, Manchester, United Kingdom; ${ }^{3}$ Department of Paediatric and Adolescent Rheumatology, Royal Manchester Children's Hospital, Manchester University NHS Foundation Trust, Manchester, United Kingdom; ${ }^{4}$ Division of Developmental and Behavioral Sciences, Section of Psychology, Children's Mercy Kansas City, Kansas City, United States of America; ${ }^{5}$ Manchester Centre for Health Psychology, Division of Psychology and Mental Health, University of Manchester, Manchester, United Kingdom; ${ }^{6}$ Division of Musculoskeletal and Dermatological Sciences, Faculty of Biology, Medicine and Health, University of Manchester, Manchester, United Kingdom

Background: Complex pain symptoms present across a wide spectrum of longterm musculoskeletal conditions in paediatric rheumatology. Pain training should therefore be part of a core curriculum for the professionals working in this speciality, but it is unclear to what extent this is the case currently.

Objectives: To identify the extent of pain-specific content included in the training of healthcare professionals in paediatric and adolescent rheumatology in the UK Methods: A systematic search of documental data using key internet search engines was conducted using combinations of the following terms: 'training', 'curriculum,' 'competency,' 'paediatric', 'adolescent' and 'rheumatology'. A targeted search of online content from the main professional organisations followed; doctors (Royal College of Paediatric and Child Health [RCPCH]), nurses (Royal College of Nursing [RCN]), physiotherapists (Chartered Society of Physiotherapy), occupational therapists (Royal College of Occupational Therapists) and psychologists (British Psychological Society). Documents from professional learned societies such as The British Society of Paediatric and Adolescent Rheumatology (BSPAR) and The Scottish Paediatric and Adolescent Rheumatology Network (SPARN) were also included. Document search strategies were designed by the authors in partnership with healthcare professionals. Data were extracted and analysed following a summative content analysis. Pain-related terms were quantified. Latent content was interpreted qualitatively to explore the context in which pain-related phrases were presented.

Results: Nine documents were identified. Pain-related terms represented $0.17 \%$ of all words across texts (used 55 times in total). Most pain terms were found in documents aimed at doctors $(n=40,72.7 \%)$. Of the pain terms used, most were used in the context of referring to specific pain syndromes such as chronic regional pain, generalized idiopathic pain and pain amplification. Content around 
the assessment and management of pain was vague and no detail was given as to how health professionals should perform these tasks. There was no reference to pain intensity, location or emotion. There were several problematic conceptual issues in the way pain was presented, with pain mostly portrayed either in the context of inflammatory or non-inflammatory pain and rarely in the context of both. Musculoskeletal pain was also positioned as a 'somatic' symptom, potentially conveying an interpretation of pain as being psychologically mediated. Conclusion: Training for healthcare professionals in paediatric rheumatology would benefit from updates informed by contemporary pain theories and evidence-based practices. This is key to ensuring that children and young people with chronic pain receive effective pain care from tertiary care services focused on treating musculoskeletal disease.

Table 1. Documents and pain terms identified

\begin{tabular}{|c|c|c|c|}
\hline Profession & Title & $\begin{array}{l}\text { Organisations, } \\
\text { year. }\end{array}$ & $\begin{array}{l}\% \text { of document } \\
\text { covered by } \\
\text { pain terms }\end{array}$ \\
\hline $\begin{array}{l}\text { Doctors } \\
\text { Doctors } \\
\text { Doctors } \\
\text { Doctors } \\
\text { Doctors }\end{array}$ & $\begin{array}{l}\text { Generic syllabus level } 1 . \\
\text { Generic syllabus level } 2 \text {. } \\
\text { Generic syllabus level } 3 \text {. } \\
\text { Paediatric rheumatology level } 3 \text {. } \\
\text { Competencies for the special interest mod- } \\
\quad \text { ule in paediatric rheumatology }\end{array}$ & $\begin{array}{l}\text { RCPCH, } 2018 . \\
\text { RCPCH, } 2018 . \\
\text { RCPCH, } 2018 . \\
\text { RCPCH, } 2018 . \\
\text { RCPCH, } 2014 .\end{array}$ & $\begin{array}{l}0.14 \% \\
0.14 \% \\
0.06 \% \\
0.48 \% \\
0.43 \%\end{array}$ \\
\hline $\begin{array}{l}\text { Nurses } \\
\text { Nurses }\end{array}$ & $\begin{array}{l}\text { Competencies for rheumatology nurses. } \\
\text { Competencies for clinical nurse specialists/ } \\
\text { advanced nurse practitioners. }\end{array}$ & $\begin{array}{l}\text { RCN, } 2020 . \\
\text { BSPAR, } 2014 .\end{array}$ & $\begin{array}{l}0.05 \% \\
0.29 \%\end{array}$ \\
\hline $\begin{array}{l}\text { Nurses } \\
\text { Allied Health } \\
\text { Professionals } \\
\text { (AHPs) }\end{array}$ & $\begin{array}{l}\text { Role of the paediatric rheumatology nurse. } \\
\text { Competencies for AHPs }\end{array}$ & $\begin{array}{l}\text { SPARN, } 2016 . \\
\text { BSPAR, } 2019 .\end{array}$ & $\begin{array}{l}0 \% \\
0.73 \%\end{array}$ \\
\hline
\end{tabular}

Disclosure of Interests: None declared

DOI: 10.1136/annrheumdis-2021-eular.210

\section{POS1491-HPR MENTAL HEALTH OUTCOMES AMONG HEALTH CARE WORKERS DURING THE CORONAVIRUS-19 PANDEMIC}

O. Hamdi ${ }^{1}$, M. Sellami ${ }^{1}$, S. Miladi ${ }^{1}$, A. Fazaa ${ }^{1}$, L. Souabni ${ }^{1}$, K. Ouenniche $^{1}$, S. Kassab ${ }^{1}$, S. Chekilii', K. Ben Abdelghani ${ }^{1}$, A. Laatar ${ }^{1} .{ }^{1}$ Mongi Slim Hospital, Rheumatology, Tunisia, Tunisia

Background: The coronavirus-19 (COVID-19) pandemic is having negative effects on societies' mental health, particularly health care workers who are exposed to tremendous psychological stress.

Objectives: To assess the magnitude of mental health outcomes among health care workers treating patients exposed to COVID-19.

Methods: This cross-sectional study collected demographic data and mental health measurements from health workers in different hospitals using an online questionnaire. Participants were also asked to complete the 9-item Patient Health Questionnaire (PHQ-9), the 7-item Insomnia Severity Index (ISI), and the 7-item Generalized Anxiety Disorder scale (GAD-7). The total scores of these measurement tools were interpreted as follows: PHQ-9 normal (0-4), mild (5-9), moderate (10-14), and severe (15-21) depression; ISI normal (0-7), mild (8-14), moderate (15-21), and severe (22-28) insomnia; GAD-7 normal (0-4), mild (5-9), moderate (10-14), and severe (15-21) anxiety.

Results: A total of 155 health care workers with a mean age of $31.3 \pm 25$ years [26-45] and a sex-ratio of 0.3 completed the online questionnaire. All participants were directly engaged in diagnosing, treating, or caring for patients with or suspected to have COVID-19. Participants were divided into two groups: $79 \%$ medical $61 \%$ doctors in training and $18 \%$ hospital doctors) and $21 \%$ paramedical staff. Twenty-two participants (14.2\%) had a chronic disease and $21(13.5 \%)$ had a history of depression. Nineteen (12.2\%) of the participants were infected with COVID-19, 4 of whom contracted the infection in the hospital $50 \%$ during October 2020). A statement to the department of occupational medicine was made in $70 \%$ of cases. Ninety-one percent of the participants were fit for treating patients with COVID-19. The mean number of nightshifts in the COVID unit per month was 5.3. The mean number of hours of work in the COVID unit was 5 hours per day, 36 hours per week, and 62 hours per month. An increase in workload compared to the pre-epidemic was noted in $62.9 \%$ of cases. Thirteen percent of participants experienced the same anxiety level as the first time of taking care of COVID patients while $65 \%$ were rather at ease compared to the first time. Forty-seven percent of participants felt the need for psychological support and $16.7 \%$ of whom had consulted a psychiatrist. Participants were diagnosed with depression (9 cases), anxiety (13 cases), and burn-out ( 3 cases). The prescribed treatment was: antidepressants (5 cases), anxiolytic (10 cases), and psychotherapy (12 cases). Mild depression was detected in $13 \%$ of cases, moderate depression in $4 \%$ of cases, and severe depression in $2 \%$ of cases. Mild insomnia was detected in $41 \%$ of cases, moderate insomnia in $14 \%$ of cases, and severe insomnia in $9 \%$ of cases. As for anxiety, $33 \%$ of participants suffered from mild anxiety, $14 \%$ moderate anxiety, and $5 \%$ severe anxiety.

Conclusion: Participants reported experiencing psychological burden and a high rate of anxiety, depression, and insomnia. It is important to protect health care workers and to promote mental well-being to survive this pandemic.

Disclosure of Interests: None declared

DOI: 10.1136/annrheumdis-2021-eular.783

\section{POS1492-HPR EVALUATION OF A VIRTUAL REALITY-BASED APPLICATION TO EDUCATE HEALTHCARE PROFESSIONALS AND MEDICAL STUDENTS ABOUT INFLAMMATORY ARTHRITIS}

P. Klemm 1 , A. Kleyer ${ }^{2}$, K. Tascilar², L. Schuster ${ }^{2}$, T. Meinderink², F. Steiger ${ }^{2}$, U. Lange ${ }^{1}$, U. Müller-Ladner ${ }^{1}$, J. Knitza ${ }^{2}$, P. Sewerin ${ }^{3}$, J. Mucke ${ }^{3}$, A. Pfeil ${ }^{4}$, G. Schett ${ }^{2}$, F. Hartmann ${ }^{2}$, A. Hueber ${ }^{5}$, D. Simon ${ }^{2}{ }^{1}{ }^{3}$ Justus-Liebig University Gießen, Campus Kerckhoff, Department of Rheumatology, Immunology, Osteology and Physical Medicine, Gießen, Germany; ${ }^{2}$ Universitätsklinikum Erlangen, Department of Internal Medicine 3, Rheumatology and Immunology, Erlangen, Germany, Erlangen, Germany; ${ }^{3}$ Heinrich Heine University Düsseldorf, Department and Hiller Research Unit for Rheumatology, Düsseldorf, Germany; ${ }^{4}$ Jena University Hospital - Friedrich Schiller University, Department of Internal Medicine 3, Jena, Germany; ${ }^{5}$ Sozialstiftung Bamberg, Sektion Rheumatologie, Bamberg, Germany

Background: Inflammatory arthritides (IA), such as rheumatoid arthritis or psoriatic arthritis, are disorders that can be difficult to comprehend for health professionals and students in terms of the heterogeneity of clinical symptoms and pathologies. New didactic approaches using innovative technologies such as Virtual Reality (VR) applications could be helpful to demonstrate disease manifestations as well as joint pathologies in a more comprehensive way. However the potential of using a VR education concept in IA has not yet been evaluated. Objectives: We evaluated the feasibility of a VR application to educate healthcare professionals and medical students about IA.

Methods: We developed a VR application using IA patients data as well as two- and three-dimensional visualized pathological joints from X-ray and computed tomography generated images (1). This VR application (called Rheumality) allows the user to interact with representative arthritic joint and bone pathologies of IA patients (Figure $1 \mathrm{~A}, \mathrm{~B}$ ). In a consensus meeting an online questionnaire was designed to collect basic demographic data (age, sex), profession of the participants, and their feedback on the general impression, knowledge gain and potential areas of application of the VR application. The VR application was subsequently tested and evaluated by healthcare professionals (physicians, researchers, and other healthcare professionals) and medical students at predefined events (two annual rheumatology conferences and academic teaching seminars at two sites in Germany). Results: 125 individuals participated in this study (56\% female, $43 \%$ male, $1 \%$ non-binary). $59 \%$ of the participants were between $18-30$ years of age, $18 \%$ between $31-40$, $10 \%$ between $41-50,8 \%$ between $51-60$ and $5 \%$ were between $61-70$. Of the participants, 50 were physicians, five researchers and four other health care professionals, the remaining were medical students (66). The participants rated the application as excellent (Figure 1 C, D), the mean rating of the VR application was 9.0/10 (SD 1.2) and many participants would recommend the use of the application, with a mean recommendation score of 3.2/4 (SD 1.1). A large majority stated that the presentation of pathological bone formation improves the understanding of the disease (120 out of $125(96 \%))$.

Conclusion: The data show that IA-targeting innovative teaching approaches based on VR technology are feasible. The use of VR applications enables a disease-specific knowledge visualization and may add a new educational pillar to conventional educational approaches.

REFERENCES:

[1] Kleyer A et al. Z Rheumatol 78, 112-115 (2019)

A
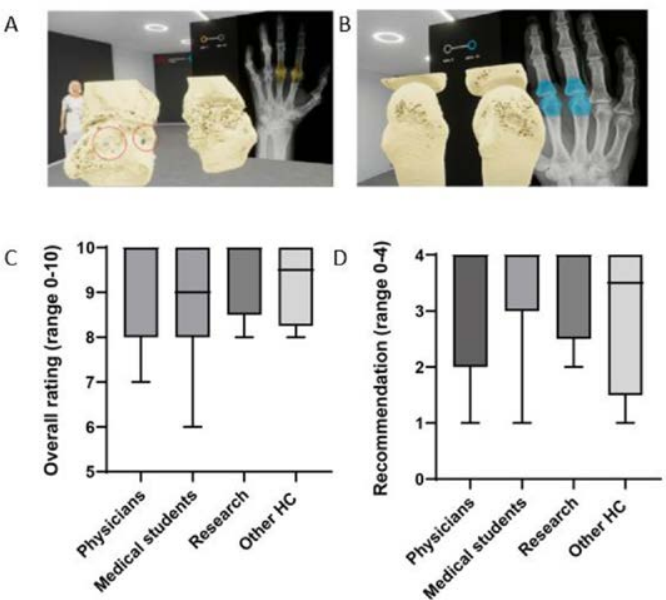

Figure 1. Illustration of the VR application and evaluation results 